\title{
Panel Regression Models for Measuring Multidimensional Poverty Dynamics
}

\author{
Gianni Betti, Antonella D'Agostino, Laura Neri \\ Dipartimento Metodi Quantitativi, Università di Siena
}

Summary: This work is related to the studying of poverty dynamics and the analysis of the socio-demographic factors influencing it. We have chosen a fuzzy and multidimmensional approach in order to define two different poverty measures. A panel regression model has been estimated and particular attention has been paid to the treatment of the unobservable heterogeneity among longitudial units. The specified model combine autoregression with variance components. The empirical analysis has been conducted using the data set of the British Household Panel Survey from 1991 to 1997.

Keywords: poverty dynamics, multidimensional measures, longitudinal units, panel regression models, unobserved heterogeneity.

\section{$1 \quad$ Introduction $^{1}$}

In the last decades many studies have paid increasing attention on the mutidimensional aspects of the phenomenom of poverty and living condition. These aspects are not taken into account in the so called traditional approach to poverty analysis that only considers monetary indicators (i.e. income or consumption expenditure); in this context the theory of fuzzy sets has been introduced by Cerioli and Zani (1990) and developed by Cheli and Lemmi

\footnotetext{
${ }^{1}$ This work was co-financed by Murst funds for the projects "Occupazione e disoccupazione in Italia: misura e analisi dei comportamenti". The paper is the result of the common work of all the authors; in particular G. Betti has written sections 2, 5.1 and 5.3.1; A. D'Agostino has written sections 4, 5.2 and 5.4; L. Neri has written sections 1, 3, 5.3.2 and 6.
} 
(1995) in order to overcome some limitations of the traditional approach and in order to define multidimensional fuzzy poverty measures. In this paper we adopt the fuzzy approach because we believe that relative well-being of individuals and/or households is a matter of degree; for this reason the division of the population into the dichotomy of the poor and the non poor seems to be an over simplification, since poverty is not a simple attribute that characterises an individual in terms of its presence or absence.

The main contribution of the paper itself consists of the comparision of panel regression models based on monetary indicators and are based on supplementary variables in order to study poverty dynamics and sociodemographic factors influecing it.

Since we concentrated out attention on poverty dynamics, we point out how fuzzy measures can overcome a further limitation of the traditional approach: the mobility of the units near to the poverty line is overestimated. Finally, another aspect to be discussed is that in the context of poverty dynamics analysis there is no unanimity in the choice of the longitudinal units; the controversy is about choosing individuals or households. In this work, the household has been chosen as a unit of analysis. Therefore this paper proposes a set of following rules that allows us to observe the dynamics of household according to the concept of poverty.

The paper is organised in the following way. In section ?? two different measures for the definition of the concept of poverty are presented. Section 3 discusses on the longitudinal units of analysis. The panel regression models are presented in section 4 . The empirical analysis, reported in section 5, is based on the data set collected by the BHPS from 1991 to 1997; finally some concluding remarks end the paper in section 6 .

\section{Poverty definitions}

The adoption of a multidimensional approach involves two main problems: the choice of the indicators and the aggregation process. Although deprivation is widely recognised as a multidimensional phenomenon, we still believe that indicators based on monetary variables have a fundamental role and therefore are worths of special treatment. For this reason two different fuzzy measures are considered: the first one is based only on a monetary variable and here it is referred to as Fuzzy Monetary (FM); the second measure is based on several indicators related to housing conditions, durable goods, 
etc... and here it is referred to as Fuzzy Supplementary (FS).

The monetary variable utilised for the FM method consists in the net equivalent household income $z_{j t}$; making use of the concepts of the fuzzy set theory, the degree of deprivation of any household $i$ at any period $t$ is defined as the membership function to the fuzzy set of poor:

$$
\mu\left(z_{i t}\right)=\left[1-F\left(z_{i t}\right)\right]^{\alpha_{t}}=I_{i t}^{F M} \quad i=1, \ldots, N t=0,1, \ldots, T
$$

where $F($.$) is the household cumulative distribution function according to the$ equivalent income. As proposed by Cheli and Lemmi (1995) we determine parameters $\alpha_{t}$ so that the membership function means are not merely equal to 0.5 , but are equal to the proportion of poor units according to the traditional approach (the so called head count ratio $H$ ). In order to identify the yearby-year household head count ratios $H_{t}$, the poverty line is calculated for the first period only and is kept fixed (in real terms) for the following years.

The FS measure is based on some supplementary variables $x_{i t k}(k=1, \ldots, K)$, such as amenities in the household, ability to afford durable goods, accommodation problems, and any other variable relevant for the multidimensional definition of deprivation. The construction process of this measure is fully described in Betti and Verma (1999). When supplementary variables are ordinal with two or more categories, for each variable $k$, with ordered categories 1 (least deprived) to M (most deprived), we define the single poverty indicator for all households in category $m$ as follows:

$$
s_{i t k}=\frac{m-1}{M-1}
$$

When supplementary variables are quantitative poverty indicators they can be calculated in a way similar to equation (1). The aggregation process of the single indicators into the multidimensional measure is described by a weighted mean:

$$
s_{i t}=\frac{\sum_{k=1}^{K} w_{k} \cdot s_{i t k}}{\sum_{k=1}^{K} w_{k}}=I_{i t}^{F S}
$$

The weights $w_{k}$ are determined by two statistical considerations: i) firstly, the weight is determined by the variable's power to "discriminate" among 
individuals in the population, that is, by its dispersion; ii) from a nonredundant point of view, it is necessary to limit the influence of those characteristics that are highly correlated to the others. For a detailed description of the weights see Betti and Verma (1999).

\section{Discussion on longitudinal statistical unit}

In the context of analysis of poverty dynamics there is no unanimity in the choice of the longitudinal unit; the controversy is about choosing individuals or households. In fact the identification of a dynamic unit can become difficult when these are complex units, such as households. Persons remain identifiable over periods, but the identification of families is complicated by marriages, divorces, births and deaths of its individual members (Duncan,1984; Trivellato, 1998). For this reason the most mature household panel survey such as PSID, BHPS identify as 'unit of analysis' the individual person, not the family or the household and establish such rule independently on the phenomenon to study. This is in contrast with cross-sectional social survey on living conditions that defines households as unit of analysis.

In fact, the concept of poverty and moreover the multidimensional concept of poverty is related to household variables rather than to individual characteristics; in the FS approach the supplementary variables consider housing conditions, the presence of some durable goods, etc...; moreover in the FM approach we consider the net 'equivalised' household income as the poverty indicator. Moreover the choice of the individual as longitudinal unit generates some heavy econometric problems in the specification of the models introduced in section 4, as foolows: i) presence of correlation among members sharing the same household across time; ii) introduction of different individual effects for units having exactly the same values for the dependent variable and the covariates.

For this reason, it is important to define a concept of longitudinal household, even if in order to follow a complex unit such as the household become more and more important the definition of a set of following rules. The rules can be simple for individuals sharing the same household across the reference period: one of these can be selected as the member representing the longitudinal unit. In the other cases, it becomes more complicated to construct rules because of longitudinal changes.

The following example can be useful to understand the problem. Let be 


\begin{tabular}{|l|l|l|l|l|}
\hline Rule & Household & $\mathbf{t}_{0}$ & $\mathrm{t}_{1}$ & $\mathrm{t}_{2}$ \\
\hline $\mathrm{A}$ & $\mathrm{i}$ & 3 & 2 & 2 \\
& $\mathrm{j}$ & & 1 & 1 \\
\hline B & $\mathrm{i}$ & 3 & 2 & 2 \\
& $\mathrm{j}$ & 3 & 1 & 1 \\
\hline C & $\mathrm{i}$ & 3 & 2 & 2 \\
\hline
\end{tabular}

Table 1: Alternative following rules

$t 0$ the starting time of a panel survey, the household $i$ at $t 0$ is composed of 3 people, between the first wave, $t 0$, and the second wave, $t 1$, the household has changed because of the leaving of one member. In order to consider as dynamic unit the household, different ways of following the dynamic unit/s can be defined : they are reported in Table 1. According to the A following rule, the original household $i$ after $t 0$ has split in two household, so in waves $t 1$ and $t 2$ the family $i$ is composed of three members and the new family $j$ is composed of one member. The problem is that according to this rule, we do not take into account of the origin of the new household $j$. For example, supposing that the member that between $t 0$ and $t 1$ left the original household $i$ was a son/daughter and that, as in our context, we are analysing poverty dynamics; the status of the new family $j$ and the dynamics of the status are obviously related to the original family, so according to this following rule a piece of information is not considered; anyway this could be partially overcome by the introduction of dummy variables reflecting at least the kind of household change. According to the B following rule, we consider the households as "sequences" of different individual histories. In this way we don't face the problem related to the origin of the new households; however we partially reintroduce the econometric problems encourred in the case of choosing the individual as unit of analysis. Finally, according to the $\mathrm{C}$ following rule, we consider the main household across time only. Obviously according to this rule we cannot follow the dynamic of the population and the panel sample do not remain representative of both individual and households. Moreover, in some cases, the choice of the "main" household could be arbitrary. Another problem is that in this way the household with less propensity to change have an higher probability to remain in the sample across time; a natural consequence is a more and more selected sample across the reference period. 
For the reasons described above in this paper we have chosen a set of rules summarised and semplified in the A rule.

\section{Poverty indicator model}

As $I_{i t}^{F M}$ and $I_{i t}^{F S}$ range in the interval $[0-1]$, a logit transformation is performed in order to create two variables ranging between $-\infty$ and $+\infty$; we obtain:

$$
y_{i t}^{F M}=\operatorname{logit}\left(I_{i t}^{F M}\right), \quad y_{i t}^{F S}=\operatorname{logit}\left(I_{i t}^{F S}\right) .
$$

The poverty indicator function for each indicator is assumed to be:

$$
y_{i t}^{(\bullet)}=\beta^{\prime} \mathbf{x}_{i t}+\Psi_{t}+u_{i t},
$$

where $\mathbf{x}_{i t}$ is a vector of $k$ time-varying exogenous variables observed on individual $i$ representing the effect of observed heterogeneity, $\Psi_{t}=f^{p}(t)$ is a polynomial of degree $p$ that represents the effect of time, $u_{i t}$ is the error structure and $\beta$ is a vector of $k$ unknown parameters. The error structure is of the form: $u_{i t}=\delta_{i}+\xi_{i t}$, where $\xi_{i t}$ has a first-order autoregressive structure, e.g. $\xi_{i t}=\rho \xi_{i t-1}+\eta_{i t}$. Here $\delta_{i}$ represents a random individual component distributed as $N\left(0, \sigma_{\delta}^{2}\right), \eta_{i t}$ is a purely random component i.i.d. assumed to be distributed as $N\left(0, \sigma_{\eta}^{2}\right)$ and $\rho$ is the serial correlation coefficient common to all individuals. The random variables $\delta_{i}$ and $\eta_{i t}$ are also assumed to be independent of each other and of $\mathbf{x}_{i t}$ and $\Psi_{t}$ (Lillard and Willis, 1978). The specified model in (5) combines autoregression with variances components to obtain a model allowing for both heterogeneity and autocorrelation (Anderson and Hsiao, 1981; Mansour et al., 1985; Goldstein et al., 1994). The individual component of this error structure, $\delta$, represents the effect of individual unobserved (or unobservable) heterogeneity in equation (5) and this effect is assumed to persist through the period of observation. The serial correlation term, $\rho$, represents the rate of deterioration of the effects of random shocks $\xi$ persisting for more than one year; it may also reflect the effect unobserved individual variables serially correlated, i.e., with a slow change across time. In econometric literature such a model is called serial correlation model as $y_{i t}$ is only affected by $x_{i t}$ not by $x_{i t-1}$, in other words if $\mathbf{x}$ is increased in period $t$ and then returned to its former level, the distribution of $y$ in period $t+1$ is not affected. Past $y$ is informative because it helps to predict the effect of unobservable variables which are serially correlated; this model also 
implies that $y_{i t}$ fluctuate around the equilibrium level $\left(\beta^{\prime} \mathbf{x}_{i t}+\Psi_{t}+\delta_{i}\right)$ as do the effects of unobservable variables $\left\{\xi_{i t}\right\}$ that follows a first-order autoregressive process. As studied in Anderson and Hsiao (1982) contrary to the case of the dynamic model for a single time series, the assumption concerning the initial observations plays a crucial role in interpreting the model and in devising consistent estimates. For this reason a special assumption is made regarding the distribution of the first response on each unit; this is taken to be the marginal distribution:

$$
y_{i 0} \sim N\left(\beta^{\prime} \mathbf{x}_{i 0}, \frac{\sigma_{\eta}^{2}}{1-\rho^{2}}\right) .
$$

This is stationary and does not depend on previous values. For $t>1$ we assume that the residual covariance structure is of the form:

$$
E\left(u_{i t} u_{j t^{\prime}}\right)=\left\{\begin{array}{c}
\sigma_{\delta}^{2}+\frac{\sigma_{\eta}^{2}}{1-\rho^{2}} \quad i=j t=t^{\prime} \\
\sigma_{\delta}^{2}+\rho^{S} \frac{\sigma_{\eta}^{2}}{1-\rho^{2}} i=j\left|t-t^{\prime}\right|=S>0 . \\
0 \quad i \neq j
\end{array}\right.
$$

As we are dealing with unbalanced panel data due to missing observations, the covariance structure reported in (7) presents no rows and columns which correspond to the missing observations (Jones, 1993).

\section{Empirical analysis}

The empirical analysis has been conducted using the data set of the British Household Panel Survey from 1991 to 1997 (Waves 1 to 7). The BHPS is a complex panel survey on incomes and other variables at household and individual level in Britain.

\subsection{Cross-sectional poverty indicators}

The sample used to construct the household poverty indicators (see equations (1) and (3)) consists of those households in which all eligible adults gave a full interview; in this data set the net equivalent household income is present for all individuals; missing values in the supplementary variables have been imputed using the approach adopted by Raghunathan et al. (1997). 
Table 2: Household membership function means

\begin{tabular}{cccccccc}
\hline \hline Wave & 1 & 2 & 3 & 4 & 5 & 6 & 7 \\
\hline$E\left[I_{i t}^{F M}\right]=H_{t}$ & 0.197 & 0.159 & 0.157 & 0.148 & 0.138 & 0.126 & 0.129 \\
$\alpha_{t}$ & 4.165 & 4.661 & 4.480 & 4.677 & 4.737 & 4.832 & 4.874 \\
$E\left[I_{i t}^{F S}\right]$ & 0.418 & 0.394 & 0.372 & 0.354 & 0.338 & 0.321 & 0.304 \\
$N$ & 4826 & 4556 & 4354 & 4378 & 4259 & 4372 & 4383 \\
\hline \hline
\end{tabular}

The household distribution function $F($.$) in equation (1) has been esti-$ mated parametrically, according to the Dagum model, on the basis of the net equivalent household income from the 1991 data set consisting of 4826 households. For the same reference year, the poverty line has been calculated as half of the mean net equivalent household income; the line results as being equal to $£ 135.45$ per week deflated to January 1998 prices. Table 2 reports the percentages of poor households in waves 1-7 according to the traditional approach (the head count ratios $H_{t}$ ) and the values of parameters $\alpha_{t}$ of formula (1) so that:

$$
E\left[I_{i t}^{F M}\right]=E\left[1-F\left(z_{i t}\right)\right]^{\alpha_{t}}=H_{t}
$$

Therefore the head count ratios coincide with the household membership function means calculated year-by-year. These show a descending behavior from 1991 to 1996, while there is a slight increment in the final year.

In order to evaluate the household membership functions according to the FS measure (formula (3)) several supplementary variables are considered; they refer to housing conditions and to the presence of durable goods; the exhaustive list of poverty symtoms is: house which is not owned; lack of central heating, colour TV, videorecorder, washing machine, dishwasher, home computer, CD player, microwave, car or van.

We would like to underline that the indicators reported in the previous list are not proper poverty symptoms: sometimes, it could merely be a matter of choice whether to own a car or not (especially if someone lives in Central London); therefore it would be more informative to know whether someone can afford a particular good. Unfortunately, this information is not collected by the BHPS, at least in the first waves.

Let us now analyse household means of the FS indicators; they are reported in the fourth row of Table 2: in this case we can observe a regular decrement of the indicators across seven years. 


\subsection{Model specification}

The analysis refers to the unbalanced panel of longitudinal households. The total sample size consists of 5734 household and 30527 repeated measures. The models specified in (5) have been estimated and in each model the dependent variable consists, alternatively, of one of the two poverty indicators. In order to compare results of the parameter estimation they have been standardized (variable names are LGFAST for $y_{i t}^{F M}$ and LGFAQST for $y_{i t}^{F S}$ ). The time indicator is the variable PEPI. A linear trend assumption has been made, e.g. the time dependence is specified as a polynomial of degree $p=1$. Anyway, it is important to underline that this assumption was made after different trials using polynomials of a greater degree or a non parametrically time dependence. The variables considered in the analysis refer to household characteristics. The variables referring to the household head are: a dummy variable for the gender, SEX (1 if male); the age and the age square, AGE and AGE2; two dummies for the employment status, JBSTA1 (1 if self or in paid employment) and JBSTA2 (1 if unemployed); four dummies for educational level, QUAL1 (1 if first degree or more), QUAL2 (1 if HND, HNC, teaching), QUAL3 (1 if A level), QUAL4 (1 if O level), a dummy variable for the marital status, MASTA (1 if married or in common law status). We consider also two dummies for macro regions, WEST (1 if South West, Midlands, Manchester, Merseyside and Wales); NORD (1 if Yorkshire, the Regions of North, Yorks \& Humber, Tyne \&Wear and Scotland), the reference macro region is the Southern regions (London inner and outer, the South-East, East Anglia, East Midlands). We also use two specifications for the household size SIZE and SIZE2 (size square). All variables are time-dependent. All models have been estimated by marginal likelihood estimation using the program MIXREG (Hedeker and Gibbons, 1996).

\subsection{Parameters estimates}

Maximum likelihood estimates of the parameters are reported in Tables 4. In each model the dependent variable is the poverty indicator $y_{i t}^{(\bullet)}$ : thus a positive sign for the parameter of a significant covariate, corresponds to a higher deprivation risk. let's first consider the effect of the trend. As expected, we observe a decreasing behaviour for both measures: this suggests a decreasing poverty risk from 1991 to 1997, even if we observe a more deep slope for the FS indicator. A possible explanation for this behaviour is a 
Table 3: Components of variance; autocorrelated individual component models

\begin{tabular}{lllllll}
\hline \hline & $\widehat{\sigma}_{u}^{2}$ & $\widehat{\sigma}_{\delta}^{2}$ & $\widehat{\sigma}_{\xi}^{2}$ & $\widehat{\sigma}_{\eta}^{2}$ & $\widehat{\gamma}$ & $\widehat{\rho}$ \\
FM & 0.687 & 0.3135 & 0.373 & 0.3316 & 0.456 & 0.3307 \\
FS & 0.623 & 0.3292 & 0.294 & 0.1728 & 0.528 & 0.6418 \\
\hline \hline
\end{tabular}

higher improvement of household conditions with respect to the economic condition in the reference period. Using the likelihood ratio test, all the parameters of the variance components are significantly different from zero (see Table 3). This result suggests that the effect of unobserved heterogeneity, interpreted as the effect of permanent differences among longitudinal units, plays an important role in the analysis of poverty dynamics. Autocorrelation has an evident effect as well. The main difference between the two measures consists in the autoregressive components: according to the FS measures the autocorrelation coefficients are larger than those in the FM. Although a serial correlation model with time-dependence variables cannot be interpreted as a state-dependence model (Lindsey, 1999), it is plausible that the residuals at time $t-1$ have a higher impact on actual poverty in the FS measure. This is because housing conditions and possession of durable goods are much less volatile than monetary variables. Permanent component patterns are similar in the two measures; this is plausible since permanent components capture the effect of permanent differences among longitudial units, however the effect of permanent component is higher in FS measure (0.528) then in FM measure (0.456). Differences between the residual error component are more evident; these components includes either the effect of transitory variables or measurement errors. The two components cannot be distinguished here; anyway, the higher residual error component in the FM measure can be explained by a larger incidence of measurement errors.

Let us consider now the role of the measured variables (fixed effects) in the variation of the membership function. Observing Tables 4 we note that for a subset of the covariates considered in the analysis there are more or less no differences between the measures FS and FM and the effect is the one expected. The household head age has a quadratic effect on the degree of deprivation, with a minimum at about fifty years (for the FM measure this is coherent with the life-cycle theory). The poverty indicator is lower if the head of the household is employed or self-employed. In the FM measure, the 
effect of the variable JBSTA2 is, as expected, always positive; the effect of an unemployed head of household is not so obvious according to the FS measure and it is not significant different from zero. It seems that according to the FS, unemployment does not affect material deprivation. The different effect of JBSTA2 regarding the FM and FS measure is likely to be related to the volatility of the income with respect to durable goods or housing conditions. The effect of the educational level of the household head is the same for the two measures (the degree of deprivation tends to decrease as the educational level increases), even if the coefficients are higher in absolute value for the FM measure. Heads of household married or in common status of law make the membership function smaller than other marital status; such an effect is likely to be associated with the age of the head of household and/or with more than one earner in the household.

A quadratic specification of the household size is significant according to the FS measure; the membership function decreases with the increase of the household size up to five members. Beyond five members, it seems that there are not sufficient economic resources to face the needs of the household members.

Instead of monetary deprivation generally increases as the household size increases; the quadratic specification is significant with a minimum of about 10 members. It is reasonable to think that the increasing trend of the membership function up to 10 household members is associated with the increasing number of children. However it is quite curious that beyond 10 members the membership function tends to decrease; a possible explanation could be the presence of one or more adult members in the household contributing in monetary terms. The SEX variable is always significantly different from zero and its effect is negative; that is, households headed by men are advantageous. The risk of poverty is higher in Northern and Western region than in Eastern regions. In particular, it seems that the difference is more deeply between Western regions than the Northern ones for both measures. The only difference is that the effects are stronger in the FS approch. This results can be explained by the evidence that differences across macro regions are concentrated in durable good and less in economic resources.

\section{Some final remarks}


Table 4: Marginal Maximum Likelihood estimates: South-Eastern Region

\begin{tabular}{lcccc}
\hline & FM measure & & FS measure & \\
\hline Variables & Estimates (s.e) & & Estimates (s.e) & \\
\hline Fixed effects & & & & \\
\hline INTERCEPT & $0.8955(0.044)$ & $* * *$ & $2.4632(0.039)$ & $* * *$ \\
PEPI & $-0.0396(0.002)$ & $* * *$ & $-0.0731(0.002)$ & $* * *$ \\
SEX & $-0.0616(0.016)$ & $* * *$ & $-0.0350(0.012)$ & $*$ \\
AGE & $-0.0236(0.002)$ & $* * *$ & $-0.0604(0.002)$ & $* * *$ \\
AGE2 & $0.0002(0.000)$ & $* * *$ & $0.0006(0.000)$ & $* * *$ \\
JBSTA1 & $-0.5131(0.015)$ & $* * *$ & $-0.1817(0.011)$ & $* * *$ \\
JBSTA2 & $0.2504(0.000)$ & $* * *$ & $-0.0163(0.015)$ & \\
QUAL1 & $-0.7282(0.026)$ & $* * *$ & $-0.3351(0.022)$ & $* * *$ \\
QUAL2 & $-0.4524(0.029)$ & $* * *$ & $-0.3310(0.024)$ & $* * *$ \\
QUAL3 & $-0.2108(0.021)$ & $* * *$ & $-0.1503(0.017)$ & $* * *$ \\
QUAL4 & $-0.1603(0.019)$ & $* * *$ & $-0.1334(0.015)$ & $* * *$ \\
MASTA & $-0.2834(0.0178)$ & $* * *$ & $-0.3045(0.014)$ & $* * *$ \\
WEST & $0.1847(0.019)$ & $* * *$ & $0.2089(0.018)$ & $* * *$ \\
NORD & $0.1778(0.019)$ & $* * *$ & $0.1479(0.019)$ & $* * *$ \\
SIZE & $0.1881(0.020)$ & $* * *$ & $-0.3095(0.016)$ & $* * *$ \\
SIZE2 & $-0.0090(0.0029)$ & $* * *$ & $-0.0260(0.002)$ & $* * *$ \\
\hline$\widehat{\rho}$ & $0.3307(0.008)$ & $* * *$ & $0.6418(0.008)$ & $* * *$ \\
\hline Random effect & & & & \\
\hline$\widehat{\sigma}_{\delta}^{2}$ & $0.3135(0.009)$ & $* * *$ & $0.3292(0.014)$ & $* * *$ \\
\hline$\widehat{\sigma}_{\eta}^{2}$ & $0.3316(0.003)$ & $* * *$ & $0.1728(0.001)$ & $* * *$ \\
\hline logL= & -30863.943 & & -21909.465 & \\
\hline & & & &
\end{tabular}


In conclusion, the definition of a set of rule for following the household in a longitudinal way has allows us to be coherent with the concept of poverty in a dynamic context. The specified models have been useful for explaining the dynamics of poverty in UK and have pointed out the presence of unobserved heterogeneity and autocorrelation. Concerning the comparison between the two measures (FM and FS) interesting results suggest that the FS measure can be used to complement the picture of poverty dynamics, and the simultaneous use of the two measures can help to better comprehend the phenomenon of deprivation.

¿From a methodological point of view, it can be added that the model specified can be generalised; for instance some of the hypotheses made, such as stationarity and independence between unobserved heterogeneity and covariates, can be relaxed. Another interesting issue to be analysed relates to the interdependence between the individual labour force process and the poverty process; in fact, in our analysis, we assume that labour force status affects the poverty condition and perhaps this assumption could be considered quite strong. It is also important to remark that it would be interesting to consider dummy variables for household changes such as covariate in the model, since these changes could influence the poverty process.

\section{References}

Anderson T.W., Hsiao C. (1981), Estimation of dynamic models with error components, Journal of the American Statistical Association, 76, 598-606.

Anderson T.W., Hsiao C. (1982), Formulation and Estimation of Dynamic Models Using Panel Data, Journal of Econometrics, 18, 47-82.

Betti G., Verma V. (1999), Measuring the degree of poverty in a dynamic and comparative context: a multi-dimensional approach using fuzzy set theory, Proceedings of the ICCS-VI, Lahore, Pakistan, August 27-31, 1999, Vol. 11, pp. 289-301.

Bryk A. S., Rudenbush S.W. (1992), Hierarchical linear models: Applications and data analysis methods, Newbury Park, CA: Sage Publications, Inc. 
Cerioli A., Zani S. (1990), A Fuzzy Approach to the Measurement of Poverty, in Dagum C., Zenga M. (eds.), Income and Wealth Distribution, Inequality and Poverty, (proc. Pavia, Italy), Studies in Contemporary Economics, Springer Verlag, Berlin, 272-284.

Cheli B., Lemmi A. (1995), A Totally Fuzzy and Relative Approach to the Multidimensional Analysis of Poverty, Economic Notes, 24, 115-134.

Duncan G.J., Coe R.D., Hill M.S. (1984), The dynamics of poverty, in Duncan D.J., Coe R.D., Corcoran M.E., Hill M.S., Hoffman S.D., Morgan J.N. (eds.), Years of Poverty, Years of Plenty: the Changing Economic Fortunes of American Workers and Families, Institute for Social Research, University of Michigan, Ann Arbor.

Goldstein H., Healy M.J.R., Rasbash J. (1994), Multilevel Time Series Models with Applications to Repeated Measures Data, Statistics in Medicine, 13, 1643-1655.

Hedeker D., GibBons R.D. (1996), MIXREG: a computer program for mixed-effects regression analysis with autocorrelated errors, Computer Methods and Program in Biomedicine.

Jones R.H. (1993), Longitudinal Data with Serial Correlation: A statespace Approach, Chapman \& Hall.

Lillard L.A., Willis R.J. (1978), Dynamic Aspect of Earning Mobility, Econometrica, 46, 985-1011.

Mansour H., Norheim E.V., Rutledge J.J. (1985), Maximum Likelihood Estimation of Variance Components in Repeated Measure Designs Assuming Autoregressive Errors, Biometrics, 41, 287-294.

Raghunathan T.E., Lepkowski J., Van Voewyk J., Solenberger P. (1997), A Multivariate Techinique for Imputing Missing Values Using a Sequence of Regression Models, Technical Report, Survey Methodology Program, Survey Research Center ISR, University of Michigan.

SniJders A.B., Bosker R.J. (1999), Multilevel Analysis: An introduction to basic and advanced multilevel modeling, Sage Publications: London. 
Trivellato U. (1998), Il monitoraggio della povertà e della sua dinamica: questioni di misura e evidenze empiriche, Statistica, 58, pp. 549-575.

Wolter K.M. (1985), Introduction to Variance Estimation, Springer Series in Statistics. 